\title{
L'avenir de la physique des hautes énergies
}

Dans le cadre du $40^{\mathrm{e}}$ anniversaire du Laboratoire de Physique Théorique (LPT) de l'École normale supérieure, une table ronde a réuni à Paris, le 22 janvier 2015, plusieurs physiciens éminents sur le thème "Quel avenir pour la physique des hautes énergies ? ".

Les participants en étaient François Englert (Université Libre de Bruxelles, prix Nobel 2013), Sheldon Glashow (Université de Boston, prix Nobel 1979), David Gross (KITP, Santa Barbara, prix Nobel 2004), Gerard 't Hooft (Université d'Utrecht, prix Nobel 1999), Giorgio Parisi (Université de Rome, médaille Boltzmann 1992) et Alexander Polyakov (Université de Princeton, médaille de Dirac 1986). Le modérateur était Lars Brink, ancien président du comité Nobel de physique.

L'intégralité du débat peut être visionnée sur le site http://savoirs.ens.fr/expose.php?id=1999 .

[*] Voir l'article de L. di Ciaccio et G. Hamel de Monchenault, p. 4 de ce numéro.

[**] Le LHC, à nouveau en fonctionnement, a produit ses premières collisions à $13 \mathrm{TeV}$ en mai 2015
Le Modèle Standard décrit avec une très grande précision les interactions fondamentales entre particules élémentaires. La dernière pierre angulaire de cette théorie, le mécanisme de brisure de symétrie de Brout-Englert-Higgs appliqué à la différentiation entre interactions faible et électromagnétique, vient d'être mise en évidence au CERN avec la découverte du très fameux "boson de Higgs " [*]. La physique des particules sera-t-elle victime de son succès ? Que faire si la deuxième phase d'exploitation $\mathrm{du}$ grand collisionneur hadronique, le Large Hadron Collider (LHC) du CERN [ $\left.{ }^{\star \star}\right]$, n'apporte pas de nouvel élément au-delà du Modèle Standard?

Les réponses des participants étaient très diverses. Shelly Glashow a insisté sur le fait qu'on ne pourra pas progresser sans l'apport de nouvelles découvertes expérimentales. Celles-ci peuvent venir de la mise en évidence de phénomènes rares (tels que la radioactivité associée à une "double désintégration bêta " sans émission de neutrinos), ou de la découverte directe de la matière sombre de notre Univers, ou encore d'un éventuel futur accélérateur opérant à une énergie cinq à dix fois supérieure à celle du LHC (qui est actuellement de $13 \mathrm{TeV}$ ). David Gross, tout en étant plus optimiste sur la phase $2 \mathrm{du}$ LHC, a fait remarquer que la faisabilité d'un tel collisionneur avait été déjà démontrée, faisant référence au projet américain du Superconducting Super Collider, avorté en 1993 pour des raisons budgétaires.

Mais est-on certain que gagner un facteur dix en énergie permettrait de découvrir la nouvelle physique qui pourrait ou devrait exister au-delà du Modèle Standard ? David Gross note que dans le cas des interactions fortes, il a fallu arriver à des énergies mille fois supérieures à la masse des pions pour tester le modèle des partons (quarks et gluons, constituants des nucléons) et leur propriété de liberté asymptotique. De plus, des arguments astrophysiques indiquent l'existence possible de particules de matière sombre dans la zone d'énergie de 1 à $100 \mathrm{TeV}$. Indépendamment de toute argumentation, indirecte ou théorique, si l'on a la possibilité de repousser les limites de nos connaissances il ne faut pas hésiter à le faire, le risque de non-découverte étant de toute façon inhérent à toute investigation scientifique.

François Englert remarque que ne pas trouver trace au LHC d'une supersymétrie entre bosons et fermions ne serait pas nécessairement une grande déception, les arguments en faveur de cette hypothèse lui semblant discutables. L'argument le plus fort en faveur de la supersymétrie pourrait selon lui venir de la théorie des cordes. Mais si celle-ci ne doit se manifester qu à l'échelle de Planck, absolument gigantesque $\left(10^{19} \mathrm{GeV}\right)$, toute vérification expérimentale directe risquerait d'être exclue. Gerard 't Hooft a plaidé en faveur d'un autre type de symétrie, encore plus fondamentale, qui permettrait de comprendre la physique à toutes les échelles, de même que la symétrie par translation assure que les lois de la nature sont les 

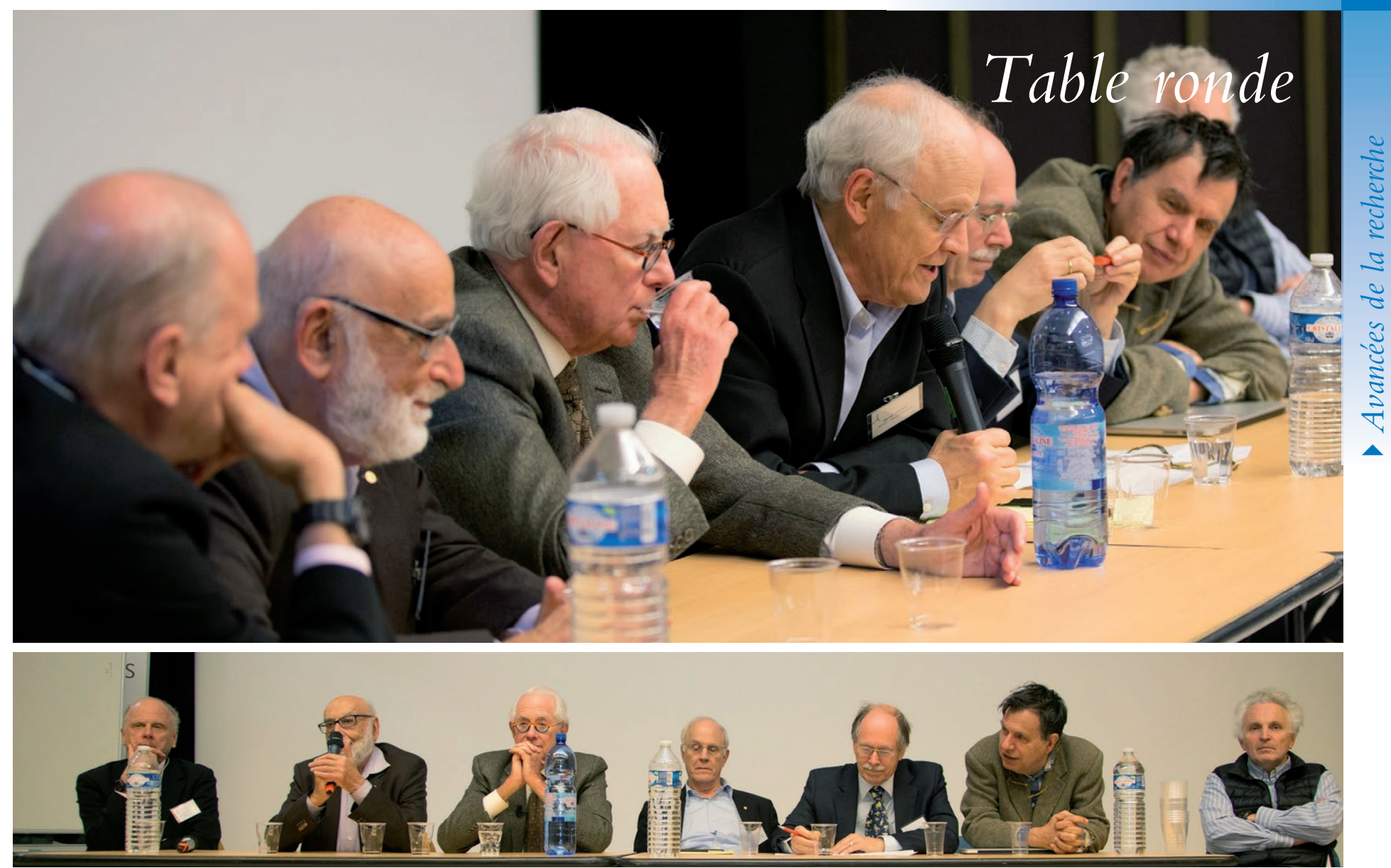

Les participants de la table ronde. De gauche à droite : Lars Brink, François Englert, Sheldon Lee Glashow, David Gross, Gerard 't Hooft, Giorgio Parisi et Alexander Polyakov. (Courtoisie : Jesper Jacobsen)

mêmes partout dans l'Univers. Il a fait référence aux années 1970, quand des percées théoriques (théories de jauge, renormalisation) ont conduit à un dénouement très rapide où tous les éléments du Modèle Standard ont été mis en place. Un tel changement de paradigme lui paraît nécessaire pour aborder la gravité quantique, qui est au centre de très nombreuses considérations théoriques.

Mais la recherche théorique peut-elle encore avancer sans attendre de nouveaux apports de l'expérience ? Sheldon Glashow n'y croit pas et remarque que ceci ne s'est produit qu'une seule fois dans toute l'histoire des sciences, quand Einstein a construit sa célèbre théorie de la Relativité Générale. Tous les autres participants ont cependant répondu par l'affirmative (mais attention, ce sont tous des théoriciens !). Sasha Polyakov, le plus optimiste, pense que la théorie quantique des champs cache encore une multitude de mécanismes et de phénomènes à découvrir. Il prend comme exemples la dualité gravité-fluide, l'instabilité associée à l'énergie sombre, ou le phénomène du confinement des quarks, encore insuffisamment compris. Giorgio Parisi ajoute qu'un grand défi théorique est d'appréhender le régime intermédiaire, ni perturbatif ni trop fort, des constantes de couplage entre particules. Il fait l'analogie avec les interactions fortes pour lesquelles, sans accélérateurs suffisamment puissants, on n'aurait pu tester la chromodynamique quantique que par des calculs numériques du spectre de masse des mésons et des baryons. Sommes-nous devant une telle situation pour la gravité quantique ? Englert, Gross et 't Hooft considèrent aussi que l'on ne pourra pas ignorer les problèmes et paradoxes impliquant la nature de l'espace-temps et la mécanique quantique, ainsi que la causalité, et que l'on devra continuer à les étudier, même à l'aide d'" expériences de pensée ".
La question inverse a aussi été débattue : la recherche expérimentale peut-elle progresser si elle n'est pas accompagnée par la théorie ? Aurait-t-on découvert le boson de Higgs si on ne savait pas ce que l'on cherchait ? L'extrême rareté des phénomènes recherchés rend leur découverte de plus en plus improbable en l'absence de guide théorique. Mais Glashow et Parisi ont aussi souligné qu'une trop grande dépendance vis-à-vis de la théorie ferait courir le risque de passer à côté d'une découverte véritablement révolutionnaire, qu'aucun théoricien n'aurait anticipé.

La physique des interactions fondamentales, ainsi que l'astrophysique et la cosmologie, sont des disciplines matures, comme l'a remarqué Lars Brink, qui doivent faire face à des contraintes sociétales et technologiques importantes. Les phénomènes physiques varient selon une échelle d'énergie logarithmique, alors que les coûts et les défis technologiques associés varient souvent de manière plus que linéaire. Qui plus est, l'impact de ces disciplines sur la vie quotidienne n'est pas le même qu'au siècle dernier.

Mais le LHC devrait nous apporter de nouveaux résultats à une énergie accrue, les recherches théoriques continuent à progresser rapidement, et les questions ouvertes sur l'unification des forces, l'origine de l'Univers et la matière sombre, et la nature quantique de l'espace-temps, restent toujours aussi fascinantes. Le débat s'est clos sur une note optimiste. Il ne faut pas sous-estimer la curiosité et l'ingéniosité des chercheurs, et l'avenir peut nous réserver bien des surprises.

Résumé du débat par Constantin Bachas (bachas@|pt.ens.fr) et Pierre Fayet Laboratoire de Physique Théorique, École normale supérieure, 24 rue Lhomond, 75231 Paris Cedex 05 